\title{
Indicadores materno-infantis na adolescência e juventude: sociodemográfico, pré-natal, parto e nascidos-vivos
}

\author{
Maternal-infantile indicators in adolescence and youth: \\ socio-demographic, prenatal, delivery and newborns \\ Maria Conceição O. Costa ${ }^{1}$, Carlos A.T. Santos ${ }^{2}$, Carlito L. Sobrinho ${ }^{3}$, \\ Juliana O. Freitas ${ }^{4}$, Karine A.S.L Ferreira ${ }^{4}$
}

\section{Resumo}

Objetivo: conhecer características e associações entre idade materna e aspectos da gestação, parto e nascidos vivos de adolescentes e adultas jovens em Feira de Santana, Bahia.

Métodos: estudo epidemiológico de corte transversal com nascidos vivos e mães adolescentes (10-16 e 17-19 anos) e adultas jovens (20-24 anos), através do Sistema de Informação de Nascidos Vivos (SINASC-1998), totalizando 5.279 nascimentos. As variáveis foram classificadas em sociodemográficas (idade, escolaridade e sexo do recém-nascido) e relacionadas a gestação, parto e condições dos recém-nascidos. Os dados foram processados usando a razão de prevalência e a análise multivariada. A regressão logística foi usada para controlar fatores de confusão (pré-natal e idade gestacional) e associar idade materna e peso de nascimento.

Resultados: em 1998, 21,6\% dos nascidos vivos no município foram filhos de adolescentes; a escolaridade de $51,2 \%$ das mães era $1^{\mathrm{o}}$ grau incompleto; a razão de prevalência e a razão de risco ajustada apontaram maior prevalência de analfabetismo, não realização do pré-natal, peso insuficiente ao nascer e menor prevalência de peso adequado, na faixa de 10 a 16 anos, comparada às demais faixas estudadas. A regressão logística mostrou associação positiva entre idade materna e peso insuficiente, bem como associação negativa com peso adequado ao nascer. Verificou-se significativa falta de registros em alguns itens do SINASC.

Conclusões: os resultados apontaram entre adolescentes alta prevalência de nascidos vivos e baixo nível de escolaridade, além do risco aumentado para analfabetismo, não realização de pré-natal, baixo peso e peso insuficiente do RN, principalmente na faixa de 10 a 16 anos, em comparação com as outras faixas estudadas.

J Pediatr (Rio J) 2001; 77 (3): 235-42: gravidez na adolescência, nascidos vivos, indicadores de saúde.

1. Doutora em Pediatria, Prof. Titular da Universidade Estadual de Feira de Santana (UEFS-Ba) e Pesquisadora do CNPq.

2. Mestre em Saúde Comunitária (Área - Estatística), Prof. Assistente da UEFS, Pesquisador do CNPq.

3. Mestre em Medicina Comunitária, Prof. Assistente da UEFS, Pesquisador do CNPq.

4. Bolsistas CNPq / PIBIC-UEFS

\section{Abstract}

Objective: to identify characteristics and associations between maternal age and gestation, delivery, and livebirths of adolescent and young adult mothers in the city of Feira de Santana, state of Bahia, Brazil.

Methods: we carried out a cross-sectional, epidemiological study with a population of livebirths and adolescent mothers (aged 10-16 and 17-19 years) and young adult mothers (aged 20-24 years). The information were obtained from the Livebirths Information System (SINASC-1998) for a total of 5,279 livebirths. Study variables were classified into socio-demographic factors (age, schooling of the mother, sex of the newborn) and related to term, delivery, gestational age, birth weight, and Apgar score. Data were processed using prevalence ratio and multivariate analysis. Logistic regression was used to control confounding factors (prenatal and gestational age) and to establish an association between maternal age and birth weight.

Results: in 1998, 21.6\% of all livebirths in the city of Feira de Santana were from adolescent mothers; out of these mothers, $51.2 \%$ had not finished junior high or elementary school. Prevalence ratio and adjusted odds ratio for the 10-16 years of age group indicated increased prevalence of illiteracy, no prenatal examination, and low birth weight and a low prevalence of adequate weight of the newborn in comparison to other age groups studied. Logistic regression showed a positive association between maternal age and low birth weight. We also observed that the SINASC database lacked information in many different fields.

Conclusions: our results indicated, for adolescent mothers, a high prevalence of livebirths and low schooling. Also, especially for the 10-16 years of age group, we observed increased risks for illiteracy, no prenatal examinations, low birth weight, and inadequate weight of the newborn in comparison to other age groups.

J Pediatr (Rio J) 2001; 77(3): 235-42: pregnancy in adolescents, health indicators, newborns.

\section{Introdução}

No Brasil, a faixa etária adolescente representa um contingente populacional considerável. Conforme dados da Comissão Nacional de População e Desenvolvimento (CNPD), em 1996, o Brasil possuía uma população de 
156,7 milhões de habitantes, dos quais 34,2 milhões eram adolescentes de 10 a 19 anos de idade ${ }^{1}$. No Município de Feira de Santana, Bahia, a faixa etária adolescente representa $24,5 \%$ (110.528) do total de 450.487 habitantes do município ${ }^{2}$.

Quanto aos aspectos relacionados à fecundidade na adolescência, sabe-se que um dos importantes determinantes do seu aumento nos últimos 30 anos diz respeito à iniciação sexual precoce nos diferentes contextos socioeconômicos e à freqüente associação deste comportamento com o desconhecimento ou conhecimento inadequado de adolescentes sobre saúde reprodutiva e anticoncepção, aliado à pouca participação da família, escolas e serviços de saúde na educação para a sexualidade. Na adolescência, a principal fonte de informação sobre sexualidade são os próprios colegas e, embora ainda estejam em plena fase de crescimento e desenvolvimento, os adolescentes têm participado de forma efetiva no aumento dos índices de partos e nascimentos, em diferentes países ${ }^{3,4}$.

No Brasil, Pesquisa Nacional sobre Saúde e Demografia, realizada pela Sociedade Civil Bem-Estar Familiar (BEMFAM), em 1996, demonstrou que, nos últimos 10 anos, a fecundidade tem diminuído em todas as faixas etárias, em torno de 30\%, com exceção da faixa adolescente. A pesquisa sobre Saúde Familiar no Nordeste, realizada pela BEMFAM, mostrou que, no Estado da Bahia, cerca de $25 \%$ das mulheres em idade fértil são adolescentes, de cada cinco adolescentes uma é considerada sexualmente ativa, sendo que $13 \%$ possuem filhos ${ }^{5,6}$.

Nos países em desenvolvimento, a gestação e a maternidade na adolescência, assim como as altas taxas de mortalidade perinatal e infanti,l encontram-se inseridas num contexto social mais amplo, conseqüência das precárias condições de vida e saúde da maioria da população. Pesquisas relacionadas à gestação e à maternidade entre adolescentes demonstram que a baixa escolaridade e a pouca profissionalização, assim como o abandono do parceiro ou da família constituem situações de risco para o comprometimento do estado de saúde da adolescente e do seu(s) dependentes(s), principalmente na ausência de suporte pré-natal ${ }^{7-9}$.

O Sistema de Informação sobre Nascidos Vivos (SINASC) faz parte do Grupo de Estatísticas Vitais do Ministério da Saúde (MS), oficializado em março de 1990. O SINASC tem como instrumento a ficha de Declaração de Nascido Vivo, que contém algumas informações sociodemográficas sobre as mães, além de outras variáveis relacionadas à gestação, parto e condições de nascidos vivos, permitindo o conhecimento do perfil epidemiológico de condições materno-infantis em diferentes localidades e possibilitando ao Sistema de Saúde viabilizar políticas e ações voltadas às reais necessidades e demandas de cada grupo populacional.

A Declaração de Nascido Vivo consta de três vias, das quais uma é encaminhada à Diretoria Regional de Saúde e as outras duas entregues à mãe para registro civil em cartório e apresentação no Serviço de Saúde na $1^{\mathrm{a}}$ consulta da criança. Compreende-se assim que o SINASC, além de fornecer dados estatísticos maternos e de nascidos vivos, fornece informações necessárias ao cartório civil para o registro da criança e ao Sistema de Saúde para matrícula de mãe e filho no Serviço. Em Feira de Santana, o SINASC foi implantado em 1996; entretanto, apenas a partir de 1998 os dados encontravam-se sistematizados de acordo com as Instituições, o que possibilitou a realização desta pesquisa para o conhecimento de indicadores de saúde do município.

O objetivo deste estudo foi conhecer as características e associações simples e múltiplas entre faixa etária materna e aspectos da gestação, parto e nascidos-vivos de adolescentes e adultas jovens em Feira de Santana, Bahia.

\section{Métodos}

Esta pesquisa consiste em um estudo epidemiológico de corte transversal da população total de nascidos-vivos e respectivas mães adolescentes de 10 a 19 anos e adultas jovens de 20 a 24 anos, no Município de Feira de Santana, em 1998, a partir de dados do SINASC-1998, totalizando 5.279 nascidos-vivos. Neste estudo, a população total foi considerada como amostra e, dessa forma, foram aplicados testes de inferência estatística, com o objetivo de generalizar os dados do município de Feira de Santana para outras localidades de características semelhantes.

A população de mães foi subdividida em grupos por faixa etária, considerando adolescentes as faixas de 10-16 anos e de 17-19 anos, e adultas jovens de 20-24 anos ${ }^{10}$. As adolescentes foram subdivididas em dois grupos, após verificar-se que o perfil da faixa até 16 anos contrastava com a faixa etária acima deste limite, conforme a literatu$\mathrm{ra}^{11-13}$.

As variáveis do estudo foram classificadas em sociodemográficas (faixa etária, escolaridade das mães e sexo dos $\mathrm{RN}$ ) e relacionadas à gestação (freqüência ao pré-natal), ao parto (tipo de parto) e às condições de nascimento (peso, idade gestacional e apgar do $5^{\circ}$ minuto de nascimento). Para a escolaridade, as mães foram classificadas em analfabeta, $1^{\circ}$ grau incompleto, $1^{\circ}$ grau completo e $2^{\circ}$ grau. A inserção ao pré-natal foi avaliada pelo número de consultas, considerando 3 categorias: não realização do pré-natal, prénatal insuficiente (com menos de 6 consultas) e pré-natal suficiente (acima de 6 consultas), segundo recomendação do Ministério da Saúde ${ }^{14}$. O parto foi classificado em 3 tipos: natural, operatório e fórceps. Quanto à idade gestacional, os RN foram classificados em prematuros $(\leq 36$ semanas); de termo ( $>36$ e $<42$ semanas) e pós-termo ( $\geq 42$ semanas), conforme classificação de Capurro ${ }^{15}$. Quanto ao peso de nascimento, os RN foram classificados em baixo peso $(\leq 2.500 \mathrm{~g})$, peso insuficiente $(2.501-3000 \mathrm{~g})$ e peso adequado (>3.000 g), conforme recomendação da OMS ${ }^{16}$. $\mathrm{O}$ índice de apgar do $5^{\circ}$ minuto de vida foi classificado por escore de pontos $<7 \mathrm{e} \geq 7^{17}$. 
Os dados foram coletados por pesquisadores treinados da equipe do NEPA, a partir da ficha de declaração de nascidos vivos do SINASC-1998 e processados nos programas EPI-INFO 6.0 e SPSSWIN 9.0.

A amostra foi descrita pelas variáveis sociodemográficas e pelas variáveis relacionadas à gestação, parto e condições de nascimento dos RN. Através da análise estatística bivariada (Razão de Prevalência-RP), obtiveram-se associações entre faixa etária materna e as outras variáveis (escolaridade, tipo de parto, realização de pré-natal, idade gestacional e peso ao nascer do RN). Para a análise multivariada utilizou-se a Regressão Logística, expressando os resultados em termos de razão de chances. Dessa forma, obteve-se a força da associação entre a variável causa (faixa etária materna) e as variáveis efeito (baixo peso, peso insuficiente e peso adequado do RN), ajustada para os fatores de confusão (realização do pré-natal e idade gestacional), mantidos no modelo ${ }^{18}$. O nível de significância estabelecido nos testes estatísticos foi de $5 \%$ ou $\mathrm{p}<0,05$.

\section{Resultados}

No presente estudo foi verificado que, do total de 10.164 nascidos vivos em Feira de Santana, no ano de 1998, 2.194 eram filhos de adolescentes e 3.085 de adultas jovens, correspondendo a $21,6 \%$ e $30,4 \%$ de nascidos vivos, respectivamente, segundo dados do SINASC-1998.

Em relação à escolaridade (Tabela 1), verificou-se que o nível mais freqüente foi o $1^{\circ}$ grau incompleto $(51,2 \%)$, com maior proporção entre adolescentes. Do total de analfabetas $(7,8 \%)$, a maior proporção ocorreu na faixa de $10 \mathrm{a}$ 16 anos. A falta de registro desta variável foi observada em $17 \%$ das fichas do SINASC.
O cálculo da razão de prevalência $(\mathrm{RP})$ de analfabetismo entre faixas etárias (Tabela 4) revelou prevalência de analfabetismo $23 \%$ e $31 \%$ maior no grupo de 10 a 16 anos em relação ao de 17 a 19 e de 20 a 24 anos, respectivamente, sem diferença estatisticamente significante. Para a escolaridade de $1^{\circ}$ grau, este mesmo cálculo apontou que a faixa de $10 \mathrm{a}$ 16 anos apresentou prevalência de $1^{\circ}$ grau incompleto $10 \%$ e $32 \%$ maior, comparada às de 17 a 19 e 20 a 24 anos, respectivamente, com diferença estatisticamente significante.

Os resultados da freqüência ao pré-natal (Tabela 2) mostraram que a maior parte das parturientes $(31 \%)$ realizou pré-natal de forma insuficiente ( $<6$ consultas) e apenas $29,6 \%$ de forma suficiente, segundo recomendações do Ministério da Saúde ${ }^{17}$. Do total de mães que não realizaram o pré-natal $(14,2 \%)$, a maior prevalência ocorreu entre adolescentes, e daquelas que realizaram de forma suficiente, a menor prevalência ocorreu na faixa de 10 a 16 anos. Ainda em relação a esta variável é importante ressaltar a ausência de registro em $25,2 \%$ das fichas do SINASC1998.

O cálculo da razão de prevalência do pré-natal apontou prevalência da não realização do pré-natal $10 \%$ maior entre adolescentes de 10 a 16, comparadas às de 17 a 19, e 32\% maior em relação às de 20 a 24 anos, com diferença estatisticamente significante (adultas jovens).

Quanto aos aspectos obstétricos, não foram verificadas diferenças na prevalência entre as faixas etárias, no que se refere ao parto natural. Este procedimento ocorreu em $78,2 \%$ do total de parturientes com prevalências semelhantes entre as três faixas etárias. Em relação à frequiência de partos operatórios $(19,2 \%)$, foi observada maior prevalência entre as adultas jovens (22\%). O parto fórceps ocorreu em apenas $0,2 \%$ das mulheres.

Tabela 1 - Distribuição de escolaridade de adolescentes e adultas jovens, segundo faixa etária, Feira de Santana, Ba, 1998

\begin{tabular}{|c|c|c|c|c|c|c|c|c|c|c|c|c|c|c|}
\hline \multirow{2}{*}{$\begin{array}{l}\text { Faixa } \\
\text { etária } \\
\text { materna }\end{array}$} & \multicolumn{2}{|c|}{ Analfab.* } & \multicolumn{2}{|c|}{$1^{\circ}$ Incomp. $\dagger$} & \multicolumn{2}{|c|}{$1^{\circ}$ Comp. } & \multicolumn{2}{|c|}{$\begin{array}{c}\text { Escolaridade } \\
2^{\circ} \mathrm{Grau}\end{array}$} & \multicolumn{2}{|c|}{ Superior } & \multicolumn{2}{|c|}{ Ignorados } & \multicolumn{2}{|c|}{ Total } \\
\hline & $\mathrm{N}^{\mathrm{o}}$ & $\%$ & $\mathrm{~N}^{\mathrm{o}}$ & $\%$ & $\mathrm{~N}^{\mathrm{o}}$ & $\%$ & $\mathrm{~N}^{\mathrm{o}}$ & $\%$ & $\mathrm{~N}^{\mathrm{o}}$ & $\%$ & $\mathrm{~N}^{\mathrm{o}}$ & $\%$ & $\mathrm{~N}^{\mathrm{o}}$ & $\%$ \\
\hline $10-16$ & 57 & 9,7 & 364 & 61,7 & 48 & 8,1 & 9 & 1,5 & - & - & 112 & 14 & 590 & 11,2 \\
\hline $17-19$ & 126 & 7,9 & 898 & 56,0 & 195 & 12,2 & 116 & 7,2 & 2 & 0,1 & 267 & 16,6 & 1.604 & 30,4 \\
\hline $20-24$ & 228 & 7,4 & 1.442 & 46,7 & 349 & 11,3 & 526 & 17,1 & 20 & 0,6 & 520 & 16,9 & 3.085 & 58,4 \\
\hline Total & 411 & 7,8 & 2.704 & 51,2 & 592 & 11,2 & 651 & 12,3 & 22 & 0,4 & 899 & 17,0 & 5.279 & 100 \\
\hline
\end{tabular}


Tabela 2 - Distribuição da freqüência ao pré-natal de adolescentes e adultas jovens segundo faixa etária materna, Feira de Santana, Ba, 1998

\begin{tabular}{|c|c|c|c|c|c|c|c|c|c|c|}
\hline \multirow{3}{*}{$\begin{array}{l}\text { Faixa etária } \\
\text { materna }\end{array}$} & \multicolumn{10}{|c|}{ Realização de consulta pré-natal } \\
\hline & \multicolumn{2}{|c|}{ Não realizado } & \multicolumn{2}{|c|}{ Insuficiente } & \multicolumn{2}{|c|}{ Suficiente } & \multicolumn{2}{|c|}{ Ignorado } & \multicolumn{2}{|c|}{ Total } \\
\hline & $\mathrm{N}^{\mathrm{o}}$ & $\%$ & $\mathrm{~N}^{\mathrm{o}}$ & $\%$ & $\mathrm{~N}^{\mathrm{o}}$ & $\%$ & $\mathrm{~N}^{\mathrm{o}}$ & $\%$ & $\mathrm{~N}^{\mathrm{o}}$ & $\%$ \\
\hline $10-16$ & 98 & 16,6 & 174 & 29,5 & 153 & 25,9 & 165 & 28,0 & 590 & 11,2 \\
\hline $17-19$ & 251 & 15,6 & 484 & 30,2 & 461 & 28,7 & 408 & 25,4 & 1.604 & 30,4 \\
\hline $20-24$ & 399 & 12,9 & 980 & 31,8 & 947 & 30,7 & 759 & 24,6 & 3.085 & 58,4 \\
\hline Total & 748 & 14,2 & 1.638 & 31,0 & 1.561 & 29,6 & 1.332 & 25,2 & 5.279 & 100,0 \\
\hline
\end{tabular}

Fonte: SINASC-1998.

Os dados do peso de nascimento (Tabela 3) apontaram $55,8 \%$ de $\mathrm{RN}$ com peso adequado $(>3000 \mathrm{~g})$, com maior prevalência entre as adultas jovens (20-24 anos). No que se refere ao baixo peso $(\leq 2500 \mathrm{~g})$ e peso insuficiente (2.501$3.000 \mathrm{~g}$ ), verificou-se que os RN de adolescentes, nas 2 faixas etárias, apresentaram maior prevalência, em relação às adultas jovens, sendo que, na faixa de 10 a 16 anos, a soma dos índices de baixo peso e de peso insuficiente $(47,3 \%)$ foi maior que o de peso adequado $(45,9 \%)$.

O cálculo da razão de prevalência do peso dos recémnascidos mostrou que os filhos de adolescentes de 10 a 16 anos apresentaram prevalência de baixo peso $9 \%$ maior em relação às de 17 a 19 anos e $33 \%$ em relação às de 20 a 24 anos (Tabela 4), sem diferença estatisticamente significante. Este mesmo cálculo para peso insuficiente revelou prevalência desta característica $19 \%$ e $34 \%$ maior entre os RN de adolescentes na faixa de 10 a 16 anos, comparada às de 17 a 19 e 20 a 24 anos, respectivamente, com diferença estatisticamente significante.
$\mathrm{O}$ resultado relacionado à idade gestacional apontou que $86,9 \%$ dos RN eram de termo, com prevalências semelhantes entre as três faixas etárias. A Razão de Prevalência para a prematuridade apontou prevalência desta característica 5\% e 7\% maior entre os RN da faixa de 10 a 16 anos, comparada às de 17 a 19 e 20 a 24 anos, respectivamente, sem diferença estatisticamente significante.

A análise multivariada do peso dos $\mathrm{RN}$ em relação às faixas etárias maternas, controlando-se fatores de confusão (pré-natal e idade gestacional) (Tabela 5), apontou prevalência de baixo peso entre RN de adolescentes de 10 a 16 $18 \%$ maior em relação às de 20 a 24 anos, sem diferença estatisticamente significante. Entretanto, para o peso insuficiente dos RN, a prevalência foi $38 \%$ maior entre os RN da faixa de 10 a 16 em relação às de 17 a 19 e de $61 \%$ em relação às de 20 a 24 anos, com diferenças estatisticamente significantes. Quanto ao peso adequado, este mesmo cálculo revelou que a prevalência desta característica foi $25 \%$ e $61 \%$ menor entre os RN da faixa de 10 a 16, comparada às

Tabela 3 - Distribuição do peso dos recém-nascidos (RN) de adolescentes e adultas jovens segundo faixa etária materna, Feira de Santana, Ba, 1998

\begin{tabular}{|c|c|c|c|c|c|c|c|c|c|c|}
\hline \multirow{3}{*}{$\begin{array}{l}\text { Faixa etária } \\
\text { materna }\end{array}$} & \multicolumn{10}{|c|}{ Peso (g) doRecém-nascido } \\
\hline & \multicolumn{2}{|c|}{$\leq 2.500 \mathrm{~g}$} & \multicolumn{2}{|c|}{$2.501-3.000 \mathrm{~g}$} & \multicolumn{2}{|c|}{$>3.000 \mathrm{~g}$} & \multicolumn{2}{|c|}{ Ignorado } & \multicolumn{2}{|c|}{ Total } \\
\hline & $\mathrm{N}^{\mathrm{o}}$ & $\%$ & $\mathrm{~N}^{\mathrm{o}}$ & $\%$ & $\mathrm{~N}^{\mathrm{o}}$ & $\%$ & $\mathrm{~N}^{\mathrm{o}}$ & $\%$ & $\mathrm{~N}^{\mathrm{o}}$ & $\%$ \\
\hline $10-16$ & 68 & 11,5 & 211 & 35,8 & 271 & 45,9 & 40 & 6,8 & 590 & 11,2 \\
\hline $17-19$ & 169 & 10,5 & 482 & 30,1 & 863 & 53,8 & 89 & 5,5 & 1.604 & 30,4 \\
\hline $20-24$ & 276 & 8,9 & 820 & 26,6 & 1.811 & 58,7 & 178 & 5,8 & 3.085 & 58,4 \\
\hline Total & 513 & 9,7 & 1.514 & 28,7 & 2.945 & 55,8 & 307 & 5,8 & 5.279 & 100 \\
\hline
\end{tabular}

Fonte: SINASC-1998. 
Tabela 4 - Razão de Prevalência (RP) de dados maternos (analfabetismo, $1^{\circ}$ grau incompleto e não realização de prénatal) e de recém-nascidos (baixo peso e peso insuficiente), segundo faixa etária materna (10 a 16 comparadas às de 17 a 19 e de 20 a 24 anos), Feira de Santana, Ba, 1998

\begin{tabular}{|c|c|c|c|c|c|c|}
\hline \multirow{2}{*}{$\begin{array}{l}\text { Faixa } \\
\text { etária } \\
\text { materna }\end{array}$} & \multirow[b]{2}{*}{ Analfabeto } & \multirow[b]{2}{*}{$\begin{array}{c}\mathbf{1}^{\circ} \text { grau } \\
\text { incompleto }\end{array}$} & \multicolumn{2}{|c|}{ Razão de Prevalência (RP) } & \multirow[b]{2}{*}{$\begin{array}{c}\text { Peso } \\
\text { insuficiente }\end{array}$} & \multirow[b]{2}{*}{ Prematuridade } \\
\hline & & & $\begin{array}{l}\text { Não realização } \\
\text { de pré-natal }\end{array}$ & Baixo peso & & \\
\hline $\begin{array}{l}10-16 \\
17-19\end{array}$ & $1,23[0,91-1,66]$ & $1,10[1,02-1,19]^{*}$ & $1,07[0,82-1,40]$ & $1,09[0,84-1,43]$ & $1,19[1,04-1,36]^{*}$ & $1,05[0,68-1,64]$ \\
\hline $\begin{array}{l}10-16 \\
20-24\end{array}$ & $1,31[0,99-1,72]$ & $1,32[1,23-1,42]^{*}$ & $1,28[1,05-1,57]^{\mathrm{a}}$ & $1,33[0,99-1,77]$ & $1,34[1,19-1,52]^{*}$ & $1,07[0,71-1,62]$ \\
\hline
\end{tabular}

* Estatisticamente significante a nível de 5\%.

de 17 a 19 e 20 a 24 anos, respectivamente, com diferenças estatisticamente significantes.

Oresultado do escore de Apgar do $5^{\circ}$ minuto de vida dos RN mostrou que $27 \%$ apresentaram escore acima de 7; entretanto, a análise e o cálculo das associações simples e múltiplas ficou prejudicada, tendo em vista que $68,2 \%$ desta variável não se encontrava registrada nas fichas do SINASC.

A distribuição dos RN por sexo mostrou $51,5 \%$ do sexo masculino e $48,4 \%$ do feminino, com proporções semelhantes entre as faixas etárias.

\section{Discussão}

A maternidade precoce tem sido identificada como um fator de afastamento e dificuldade de retorno da mãe adolescente aos estudos; entretanto, sabe-se que outros fatores sociais e econômicos podem interferir no processo de escolarização e formação profissional nos diferentes níveis socioeconômicos ${ }^{7,8,11}$.

Neste estudo, a alta prevalência $(56 \%)$ do $1^{\circ}$ grau incompleto observada entre as adolescentes de 17 a 19 anos contraria os limites etários estabelecidos pelo Ministério da Educação e Cultura-MEC: para a escolaridade de $1^{\circ}$ grau, os limites são de 7 a 14 anos, e para a de $2^{\circ}$ grau, são de 15 a 19 anos. Estes resultados concordam com os de outros estudos, que observaram índices superiores a 70\% da escolaridade de $1^{\circ}$ grau incompleto entre gestantes e mães adolescentes ${ }^{10,11,19}$. Em relação à escolaridade, ainda é importante ressaltar o alto percentual da inexistência de informações verificada na presente pesquisa, apontando a necessidade de sensibilizar profissionais de saúde quanto ao registro correto de dados nos Sistemas Vitais de Informações, os quais revelam demandas sociais e subsidiam estratégias de intervenção.
O pré-natal é amplamente reconhecido como um dos principais determinantes da evolução gestacional normal. Segundo o Programa Assistência Integral a Saúde da Mulher - PAISM (MS), a assistência pré-natal adequada deve garantir o mínimo de 6 consultas; entretanto, vale a pena ressaltar que a qualidade desta assistência inclui início precoce, acompanhamento das curvas de peso e altura uterina, avaliação periódica de parâmetros vitais, entre outros requisitos indispensáveis, os quais exigem capacitação técnica e entrosamento entre a equipe ${ }^{14,20,21}$. Os resultados desta pesquisa concordam com os de outros estudos, que verificaram alta proporção de ingresso tardio e ausência ao pré-natal entre adolescentes ${ }^{12,20,23}$.

Diferentes fatores são apontados para explicar a ausência ou inserção tardia de adolescentes ao pré-natal, entre eles destacam-se as dificuldades de assumir a gestação, conflitos familiares, assim como desconhecimento da importância desta assistência, situação que é agravada na presença de abandono da família e/ou do parceiro $4,7,8,22$.

Os resultados referentes ao parto natural, da presente pesquisa, concordam com os de outros estudos, que não apontaram diferenças quanto a esta característica entre adolescentes e adultas sob as mesmas condições de vida ${ }^{22,23}$. Algumas pesquisas verificaram índices que variam de 36 a $63 \%$ de parto vaginal entre adolescentes $8,11,24$. Em relação ao parto operatório, é importante destacar que, embora nesta pesquisa o índice tenha ultrapassado o limite considerado adequado pela OMS (15\%), este resultado está de acordo com estimativas nacionais como a Pesquisa Nacional de Demografia e Saúde, que apontou o aumento do índice de cesariana de $31,6 \%$ para $36,4 \%$ na década de 1986 a1996 ${ }^{5}$.

Em relação ao peso de nascimento, os achados deste estudo concordam com os de outras pesquisas, que verificaram maior proporção de baixo peso e de peso insuficiente 
Tabela 5 - Razão de chance ajustada*, do baixo peso, peso insuficiente e peso adequado de recém-nascido (RN), segundo faixa etária materna (10 a 16 comparadas às de 17 a 19 e 20 a 24 anos), Feira de Santana, Ba, 1998

\begin{tabular}{lccc}
\hline Faixa etária & Baixo peso & Peso insuficiente & Peso adequado \\
\hline $\mathbf{1 0}-\mathbf{1 6}$ & & & \\
$\mathbf{1 7}-\mathbf{1 9}$ & $0,97[0,68-1,38]$ & $1,38[1,12-1,17] \dagger$ & $0,75[0,61-0,92] \dagger$ \\
$\mathbf{1 0}-\mathbf{1 6}$ & & & \\
$\mathbf{2 0}-\mathbf{2 4}$ & $1,18[0,84-1,66]$ & $1,61[1,34-1,99] \dagger$ & $0,61[0,50-0,73] \dagger$ \\
\hline
\end{tabular}

* Pré-natal e idade gestacional

† Estatisticamente significante a nível de 5\%

entre RN de adolescentes na faixa etária até 16 anos. Nos EUA, Stevens-Simon \& McAnarney, $1988^{25}$, verificaram $13,8 \%$ de baixo peso entre adolescentes da faixa até 15 anos, 9,3\% na faixa de 17 a 19 e 5,8\% naquelas de 25 a 29 anos; em Campinas, SP, Bicalho-Mariotoni \& e Barros Filho, $1995^{26}$, apontaram entre RN de adolescentes, 16,6\% de baixo peso na faixa até 15 anos e $11,4 \%$ até 19 anos; em Belém, Costa et al, $1999^{11}$, verificaram que a proporção de baixo peso somado a peso insuficiente foi superior $(51,6 \%)$ à proporção de peso adequado $(48,4 \%)$, entre RN de adolescentes com idade até 16 anos.

Nas duas últimas décadas, estudos têm buscado esclarecer a interferência de diferentes fatores nos resultados neonatais. Pesquisas têm mostrado que RN de adolescentes apresentam características antropométricas semelhantes aos filhos de adultas, nas mesmas condições de vida. Entretanto, os RN de adolescentes da menor faixa etária (10 a 16 anos) apresentam maior prevalência de peso insuficiente (2.501-3.000g) e baixo peso $(\leq 2.500 \mathrm{~g})$ e menor prevalência de peso adequado $(>3.000 \mathrm{~g})$, quando comparados com os de mães adultas nas mesmas condições de vida. Entre os fatores apontados destacam-se o baixo peso materno anterior à gestação, o ganho ponderal insuficiente e as intercorrências gestacionais associadas aos conflitos familiares e com o parceiro, as quais interferem no autocuidado com a saúde ${ }^{8,28}$. Outros fatores apontados são o incompleto crescimento físico (composição corporal, órgãos da reprodução) e a baixa idade ginecológica verificada entre adolescentes da faixa até 15 anos, podendo interferir na transferência de nutrientes para o feto, pela insuficiência úteroplacentária $^{12,28,29}$.

Os resultados deste estudo concordam com dados da literatura, tanto no que se refere à prevalência de peso insuficiente e de peso adequado quanto à idade gestacional de termo e de pré-termo 9,12,14,26,28. Em Campinas, SP, dados do SINASC de $1995^{26}$ mostraram índice geral de prematuridade de $6,2 \%$, sendo 7,6\% filhos de adolescentes e, entre as mães de RN de baixo peso e prematuros, $22,8 \%$ e $25,9 \%$, respectivamente, eram adolescentes, não sendo observado risco aumentado associado à idade materna.

Em relação à variável Apgar de $5^{\circ}$ minuto, 68,2\% dos dados não apresentavam registro, o que dificultou a análise dos dados. Este resultado aponta a pouca importância dada ao preenchimento da declaração de nascidos vivos pelos profissionais de saúde, nas maternidades, atitude esta que vai de encontro ao consenso geral quanto à importância da assistência perinatal, no que se refere à diminuição dos índices de mortalidade, assim como do registro de dados vitais que, na condição de indicadores, servem de subsídios para o sistema de saúde no redimensionamento de suas práticas voltadas à melhoria da assistência materno-infantil. O preenchimento adequado do Sistema de Registro de Nascidos Vivos é fonte importante de informação sobre a saúde da gestante e do recém-nascido e sobre a assistência prestada pelos Serviços de Saúde.

A inexistência de dados aponta para a necessidade de sensibilizar os profissionais de saúde quanto à importância destes dados e quanto à interferência no processo de interpretação e análise de dados vitais que podem subsidiar políticas públicas e ações fundamentais à saúde da mãe e do filho na gestação, no parto e no puerpério.

\section{Conclusões do estudo}

Os resultados do estudo apontaram, entre adolescentes das duas faixas etárias, alta prevalência de nascidos vivos, analfabetismo, escolaridade de $1^{\circ}$ grau e não realização de pré-natal.

Os recém-nascidos de adolescentes de 10 a 16 anos apresentaram maior prevalência e chance de peso insuficiente e menor prevalência e chance de peso adequado, quando comparados aos filhos das outras faixas etárias estudadas. 


\section{Considerações sobre o método estatístico e os resultados}

Em relação ao delineamento, cabe considerar a relação de determinação, através de estudo de corte transversal; entretanto, neste estudo, a falta de dimensão temporal foi parcialmente superada pela caracterização de anterioridade da gestação em relação ao parto e ao nascido vivo.

Outro aspecto que merece consideração refere-se à consistência científica dos achados, relacionado ao controle dos fatores de confusão, realizado através da análise multivariada e que coincide com dados da literatura revisada.

\section{Considerações sobre propostas de intervenção}

São as seguintes as propostas que emanam dos resultados deste estudo:

- necessidade de sensibilização dos profissionais de saúde quanto à importância do preenchimento adequado da ficha do SINASC;

- investimento dos Serviços de Saúde em ações específicas de captação precoce e inserção da mãe adolescente ao pré-natal, acompanhamento pós-parto e planejamento familiar;

- implementação de ações de sensibilização da população adolescente pelos Sistemas de Educação e de Saúde quanto à responsabilidade de prevenir a gestação precoce e não planejada e quanto às suas consequiências.

\section{Agradecimento Especial}

Ao Prof Dr Balmukund Niljay Patel, pela valiosa contribuição na correção do abstract e sugestões no método estatístico.

\section{Referências bibliográficas}

1. Comissão Nacional de População e Desenvolvimento. Reunião Regional da América Latina. Saúde Sexual e Reprodutiva de Adolescentes. Costa Rica, Brasília: Fundo de População Nações Unidas; 1998.

2. Anuário Estatístico de Feira de Santana 1998;1:1-356.

3. Barroso C, Campos MM, Moraes MLQ. Gravidez da adolescência. Brasília: IPLAN/IPEA/UNICEF, Fundação Carlos Chagas; 1986.

4. OPAS \& UNICEF. A situação da fecundidade. Perfil estatístico de crianças e mães no Brasil. Rio de Janeiro: IBGE/UNICEF/ OPAS; 1988.
5. BEMFAM Sociedade Civil de Bem Estar Familiar. Pesquisa Nacional sobre Saúde Familiar no Nordeste. Relatório 1991/ 1992.

6. BEMFAM - Sociedade Civil de Bem Estar Familiar. Pesquisa Nacional sobre Demografia e Saúde. Rio de Janeiro: relatório BEMFAM; 1996.

7. Costa MCO, Pinho FJ, Martins SJ. Aspectos psicossociais e sexuais de gestantes adolescentes em Belém, Pará. J Pediatr (Rio J) 1995;71:151-7.

8. Guimarães MHP. Gravidez na adolescência: seus determinantes e conseqüências [dissertação]. Salvador: UFBA; 1994.

9. Organización Mundial de La Salud. Risk approach for maternal and child health care. Geneva: WHO; 1978.

10. OPAS - OMS. Fecundidad en la adolescencia: causas, riesgos y opciones. Washington DC: Cuadern Técnico no 12; 1988.

11. Costa MCO, Queiroz SS, Santos ACT, Barboni AR, Oliveira Neto AF, Rocha GR, et al. Condições de gestação, parto e nascimento em adolescentes e adultas jovens: Santa Casa Maternidade de referência do SUS, Belém, Pará. Rev Adolesc Latino Am 1999;1:242-51.

12. Frisancho AR, Matos J, Bollettino LS. Role of ginecological age and growth maturity atatus in fetal maturation and prenatal grown of infants born to young still growing adolescent mothers. Hum Biol 1984;56:583-93.

13. Maso M, Gong EJ, Jacobson MS, Bross DS, Heald FP. Anthropometric predictors of low weight outcome im teenage pregnancy. J Adolesc Health Care 1988;9:188-93.

14. BRASIL, Ministério da Saúde. Secretaria Nacional de Programas Especiais. Divisão de Saúde Materno-Infantil. Assistência Pré-natal. 2nd ed. Brasília: Manual Técnico; 1988.

15. American Academy of Pediatrics. Committee on fetus and newborn. Nomenclature for duration of gestation, bitter weight and intrauterine growth. Pediatrics 1967;39:935-9.

16. Nóbrega FJ, Brasil AL, Lopes FA. Recém-nascidos de mães adolescentes brasileiras. Antropometria e outras variáveis. International Society Pediatric Nutrition 1991;Suppl 1.

17. Apgar V. Sistema of pontuacion (Apgar) para el recién nacido. Clin Pediatr North America 1966.

18. Rosner B. Fundamentals of Biostatistics. 4th ed. United States of America: ITP; 1995.

19. Molina R, Alarcón G, Luengo X. Estudio prospectivo de factores de riesgo en adolescentes embarazadas. Rev Chil Obstet and Ginecol 1988;53:27-34.

20. Greenberg RS. The impact of prenatal care in different social groups. Am J Obstet and Gynecol 1983;145:797-801.

21. Rebolledo AG, Atalah E. Riesgos nutricinales en embarazadas adolescentes. Rev Chil 1986;14:193-8.

22. Viçosa GR, Ruzicki EM, Przybyiski J, et al. Gestação na adolescência: a experiência do Hospital Presidente Vargas. Revista de Psiquiatria do Rio Grande do Sul 1987;9:97-104.

23. Aliaga EM, Suazo EW, Diaz JI, Munõz RG, Meneses MCB, Araya MO, Venegas MEV. Experiência en una unidad de gestantes precoces. Rev Chil Obstet Ginecol 1985;127:37.

24. Mathias L. Idade limite de risco reprodutivo entre adolescentes. J Bras Ginecol 1985;95:141-3.

25. Stevens-Simon C, Mc Anarney ER. Adolescent maternal weight gain and low birth weight: a multifactorial model. Am J Clin Nutr 1988;47:948-53.

26. Bicalho-Moriotoni GGB, Barros Filho AA. Nascer em Campinas: análise dos dados do Sinasc 1995. J Pediatr (Rio J) 1998;74:107-13.

27. Vitalle MSS, Brasil ALD, Nóbrega FJ. Recém-nascido de mãe adolescente de baixo nível sócio-econômico. Rev Paul Pediatr 1997;15:17-24. 
28. School TO, Hediger ML, Ances IG, Belsky DH, Salmon RW. Rate and amount of gain during adolescent pregnancy: associations with maternal weight-for-heigth and birth weight. Am J Clin Nutr 1990;52:793-9.

29. Frisancho AR, Matos J, Leonard WR, Yeroch LA. Developmental and nutritional determinants of pregnancy outcome among teenagers. Am J Phys Anthropol 1985;66:247-61.
Endereço para correspondência:

Dra. Maria Conceição Oliveira Costa

Avenida Euclides da Cunha 475, ap.1602 - Graça

CEP 40150-120 - Salvador, Bahia

Fone/Fax: (71) 237.1584 e 235.8753

E-mail: nepa@zipmail.com.bre costamco@uol.com.br 